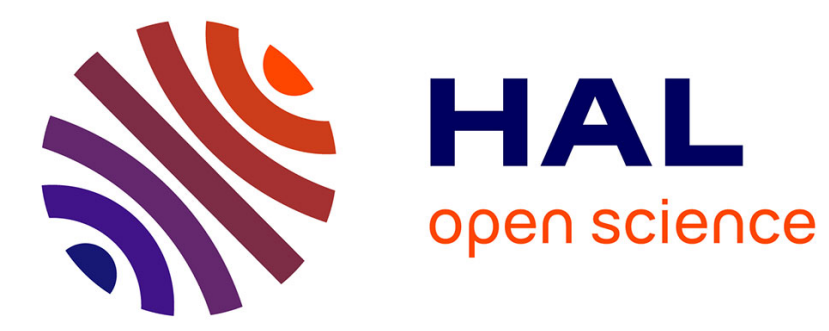

\title{
Genre et associations en Europe : le pouvoir en question
}

Erika Flahault, Anne Guardiola

\section{To cite this version:}

Erika Flahault, Anne Guardiola. Genre et associations en Europe : le pouvoir en question. Informations sociales, 2009, 151, pp.128-136. halshs-00921870

\section{HAL Id: halshs-00921870 \\ https://shs.hal.science/halshs-00921870}

Submitted on 22 Dec 2013

HAL is a multi-disciplinary open access archive for the deposit and dissemination of scientific research documents, whether they are published or not. The documents may come from teaching and research institutions in France or abroad, or from public or private research centers.
L'archive ouverte pluridisciplinaire $\mathbf{H A L}$, est destinée au dépôt et à la diffusion de documents scientifiques de niveau recherche, publiés ou non, émanant des établissements d'enseignement et de recherche français ou étrangers, des laboratoires publics ou privés. 
Flahault Érika, Guardiola Anne, 2009, « Genre et associations en Europe : le pouvoir en question », Informations sociales, $\mathrm{n}^{\circ} 151$, janvier-février, " Genre et pouvoir en Europe », pp. $128-136$

\section{Genre et associations en Europe : le pouvoir en question}

Erika Flahault - sociologue, laboratoire ESO - Espaces et Sociétés UMR CNRS 6590, Université du Maine

Anne Guardiola - sociologue, laboratoire Printemps UMR CNRS 8085 (Professions, institutions, temporalités), Université de Versailles Saint-Quentin-en-Yvelines), Union nationale Retravailler.

Les associations sont souvent perçues comme l'instrument privilégié de la participation citoyenne et le mode d'expression idéal de la société civile. La question de l'accès au pouvoir en leur sein n'est pourtant guère posée ; pas plus que n'est interrogée la distribution sexuée de ce pouvoir. L'objectif de cet article est de dresser un état des lieux de la situation des hommes et des femmes au regard du pouvoir dans les mondes associatifs des différents pays d'Europe, dans une perspective de genre. Mais un tel projet se heurte à une triple difficulté. D'une part, il n'existe pas de statut unifié des associations au niveau européen. Le paysage associatif présente une extrême diversité d'un pays à l'autre et aucun critère commun n'a été défini jusque-là pour en délimiter le périmètre. Cette hétérogénéité se reflète dans le vocabulaire utilisé : entre association sans but lucratif, organisation non gouvernementale, fondation, organisation bénévole, coopérative sociale, secteur communautaire, secteur volontaire, les nuances sont nombreuses et nous invitent à la plus grande prudence en matière de comparaison. Ensuite, les recherches sur l'économie sociale et solidaire en général, et sur les associations en particulier, sont souvent récentes et de ce fait peu développées dans certains pays. Quand elles existent, elles se fondent sur des méthodologies diverses et sont loin d'être exhaustives. En France, par exemple, les enquêtes actuelles reposent sur des sondages auprès d'un échantillon de personnes ou de grandes associations, tous secteurs confondus. On a peu d'informations, en dehors de monographies, sur les associations « locales » sans salariés, pourtant les plus nombreuses. Et dans plusieurs pays, il est encore impossible de compter sur des statistiques fiables. Enfin, la question de la place des femmes est rarement abordée. Récente, voire émergente, elle apparaît peu légitime. Lorsqu'elle est traitée, c'est à peu près toujours sur un plan général et peu sur la dimension de l'accès au pouvoir. Les résultats de ces rares analyses restent peu visibles. Jude Howell (2007) montre que les théoriciens de la « société civile ", passés et présents, ont accordé très peu d'attention à la dimension genrée de la société civile, tandis que les théoriciennes féministes se sont peu intéressées aux recherches sur l'économie sociale privilégiant l'analyse du marché, de l'Etat et de la famille. Cette 
ignorance mutuelle, qui vient du fait que les deux champs se fondent sur des bases conceptuelles différentes, expliquerait, selon elle, la rareté des données sur la question.

Pour toutes ces raisons, on déplore une absence de données statistiques complètes et exploitables sur les acteurs du monde associatif en Europe ; tant au niveau global européen qu'au niveau de la plupart des Etats membres. A titre d'exemple, les seules informations disponibles sur les sites d'Eurostat et de la Commission Européenne (rubrique Femmes et hommes dans la prise de décision) ne concernent que les ONG européennes, composées de $39 \%$ de femmes et $61 \%$ d'hommes et présidées dans $70 \%$ des cas par un homme (Eurostat, 2008).

Nous avons donc fait le choix, largement contraint, de ne traiter que de trois pays pour lesquels des informations sont disponibles, avec toute la prudence qui s'impose en raison des réserves évoquées précédemment : Espagne, France, Royaume-Uni.

\section{Panorama du monde associatif dans les trois pays étudiés}

Dans le cadre des travaux internationaux lancés en 1990 par l'Université John Hopkins (US) et la London School of Economics (RU), Edith Archambault (2007) a pu dresser une typologie, aujourd'hui classique, des cinq variantes du modèle européen des "sociétés sans but lucratif » (SSLB), dans laquelle nous pouvons replacer les trois pays retenus.

La France s'inscrit dans la variante continentale ou corporatiste. Le secteur associatif y est largement développé et partiellement professionnalisé ; tout particulièrement dans le secteur sanitaire et social où il offre une large palette de prestations de services. Les associations sans salarié y sont toutefois de loin majoritaires $(84 \%)$. Ces organisations sont fédérées, souvent selon leur appartenance idéologique, et sont financées essentiellement par l'Etat, les Collectivités territoriales et la Sécurité sociale, " conformément au principe de subsidiarité » (Archambault, 2007, p. 17) pour les associations employeuses ; principalement par les recettes d'activité et les cotisations pour les autres au premier titre desquelles on trouve les associations sportives qui représentent près d'un quart des structures associatives mais moins de 7\% de l'emploi salarié associatif (Tchernonog 2007).

Le Royaume-Uni apparaît comme l'archétype de la variante anglo-saxonne ou libérale. Les organisations associatives (voluntary organisations, charities) y sont fondées sur « une longue tradition d'initiative privée charitable qui s'exerce en relation étroite avec les collectivités locales » (Archambault, 2007, p. 18). Cette culture associative s'ancre dans une socialisation précoce au bénévolat dès le système scolaire ; et elle repose sur une forte complémentarité entre un bénévolat dynamique et un salariat très professionnalisé et très qualifié. Les financements y sont variés, avec un rôle important des fondations et de l'Etat via les bénéfices de la loterie nationale. Les évolutions récentes sont marquées par la laïcisation et par l'intégration d'une logique plus marchande, sous l'impulsion de l'Etat.

L'Espagne représente la variante méditerranéenne ou émergente. Le secteur associatif y est moins développé car longtemps interdit ou très contrôlé. L'assistance et la prestation de services sociaux y furent longtemps prises en charge par l'entraide informelle au sein de la famille, de la paroisse, du village. De 1938 à 1975, pendant la dictature de Franco, seules Caritas et ONCE, fondées respectivement par l'Eglise et par l'Etat pour l'aide aux aveugles, et la Croix-Rouge sont actives. Lors de la transition démocratique qui suit, l'Etat prend en charge les politiques sociales. Le bénévolat informel y est donc davantage développé que le bénévolat associatif. Ce dernier prend toutefois de l'ampleur à partir des années 1980-90 et se trouve encore aujourd'hui en pleine structuration. Les financements publics y sont importants. 


\section{Principaux indicateurs}

\begin{tabular}{|c|c|c|c|}
\hline & Espagne & France & Royaume-Uni \\
\hline $\begin{array}{l}\text { Nombre } \\
\text { d'associations }\end{array}$ & 125300 & $\begin{array}{l}1.100 .000 \text { dont } \\
173500 \text { employeuses }\end{array}$ & 190000 charities \\
\hline Budget & $\begin{array}{l}21,7 \text { million d'euros pour } \\
\text { le tiers secteur, en } 2001 \text {, } \\
\text { soit } 3,3 \% \text { du PIB dont } 7.3 \\
\text { millions pour le secteur } \\
\text { associatif. }\end{array}$ & $\begin{array}{l}59,4 \text { milliards d'euros soit } \\
3,5 \% \text { du PIB }\end{array}$ & $\begin{array}{l}46.3 \text { milliards d'euros } \\
\text { (35 milliards de livres) } \\
\text { soit } 4 \% \text { du PIB }\end{array}$ \\
\hline Ressources & $\begin{array}{l}\text { Pour le tiers secteur: } \\
\text { Public, } 56,6 \% \\
\text { Privé, } 41,2 \% \\
\text { Autres : } 2,3 \%\end{array}$ & $\begin{array}{l}\text { Public: } 34.3 \% \\
\text { Dons et mécénat: } 4,9 \% \\
\text { Recettes d'activité et } \\
\text { cotisations: } 60.8 \%\end{array}$ & $\begin{array}{l}\text { Public : } 47 \% \\
\text { Dons et mécénat : } 9 \% \\
\text { Recettes d'activité et } \\
\text { cotisations : } 45 \%\end{array}$ \\
\hline Bénévolat & $\begin{array}{l}1 \text { million de bénévoles, } \\
\text { dont } 253599 \text { ETP }\end{array}$ & $\begin{array}{l}14 \text { millions de bénévoles } \\
\text { actifs, dont } 935400 \text { ETP }\end{array}$ & $\begin{array}{l}22 \text { millions de bénévoles, } \\
\text { dont } 1100000 \text { ETP }\end{array}$ \\
\hline Emploi salarié & $\begin{array}{l}548 \text { 366, dont } 475179 \\
\text { ETP }\end{array}$ & $\begin{array}{l}1680000 \text {, dont } 1360000 \\
\text { ETP (INSEE 2008) à } \\
1.920 .000, \text { dont } 1046000 \\
\text { ETP (Tchernonog 2007) }\end{array}$ & $\begin{array}{l}611.000, \text { dont } 486000 \\
\text { ETP (2005) }\end{array}$ \\
\hline $\begin{array}{l}\text { Part de l'emploi } \\
\text { associatif dans } \\
\text { l'emploi total } \\
\text { salarié }\end{array}$ & $\begin{array}{l}3,9 \% \text { de la masse } \\
\text { salariale totale }\end{array}$ & $\begin{array}{l}6 \% \text { de l'ensemble des } \\
\text { emplois salariés } \\
8,5 \% \text { de la part des emplois } \\
\text { salariés du secteur privé } \\
6,4 \% \text { de la masse salariale } \\
\text { du secteur privé }\end{array}$ & $\begin{array}{l}2,2 \%(2005) \text { de } \\
\text { l'ensemble des emplois } \\
\text { rémunérés }\end{array}$ \\
\hline
\end{tabular}

Sources : Pour l'Espagne : García (2004), Lorenzo García (2003). Pour la France : CPCA (2008), Insee (2008) statistiques harmonisées de l'économie sociale, Tchernonog (2007). Pour le Royaume-Uni : CPCA (2008), Clark (2007).

Si les données de cadrage sur la vie associative sont disponibles de façon relativement homogène pour les trois pays, les données sexuées sont plus rares et généralement cantonnées à des échantillons sectoriels, géographiques ou volontaires d'associations. Ceci introduit un biais méthodologique important. Nous nous proposons néanmoins de présenter ces résultats incomplets, première étape pour susciter une réelle mise en œuvre de statistiques européennes dans le domaine.

\section{La place des femmes dans les associations... la part des bénévoles}

Au-delà des spécificités nationales pointées, la place des femmes dans les sphères bénévoles du pouvoir associatif présente des similitudes.

En France, on note une moindre participation des femmes à tous les niveaux du monde associatif. Le taux d'adhésion à au moins une association atteint $39 \%$ pour les femmes contre $49 \%$ pour les hommes. Et la participation bénévole aux activités associatives est estimée à $19 \%$ pour les femmes et $27 \%$ pour les hommes (Fèbvre \& Muller, 2004). Un examen des fonctions dirigeantes montre une constante, la prédominance masculine aux postes de pouvoir, qui se décline de façon variable selon le secteur d'activité, la taille de la structure et son ancienneté (Tchernonog, 2007). La présidence des associations reste très majoritairement masculine (69\%). Toutefois, les femmes sont un peu plus présentes parmi les présidentes d'associations employeuses (36\%) que d'associations sans salariés $(30 \%)$; de plus près, on note que ce constat est vrai dans les associations de 5 à 19 salariés mais s'inverse dans les organisations de plus de 50 salariés. On observe une situation proche de la parité dans deux secteurs d'activité : l'action caritative et humanitaire $(47 \%$ de femmes présidentes) et l'action sociale (47\%). Les recherches historiques menées par Evelyne Diebolt (2004) ont toutefois montré que les femmes avaient été progressivement éliminées des postes de décision dans le secteur sanitaire et social où elles ont été plus nombreuses lors des créations. En revanche, les secteurs Chasse et pêche, Sports et Défense des droits et 
des causes laissent très peu de place aux femmes présidentes (respectivement $3 \%, 17 \%$ et $20 \%$ ). Enfin, les présidentes apparaissent d'autant plus nombreuses que l'association est récente. Elles ne sont que $17 \%$ dans les structures créées avant 1960 mais 38\% dans les plus récentes, déclarées après 2000 .

Les femmes sont proportionnellement plus nombreuses sur les autres fonctions du bureau, puisqu'elles représentent $42 \%$ des trésoriers et $57 \%$ des secrétaires. De ce fait, une analyse globale de l'ensemble des bureaux (président, trésorier, secrétaire) laisse apparaître une quasi parité, par ailleurs peu sensible à la taille des structures. Comme le notent Viviane Tchernonog et Muriel Tabaries, « cela suggère que les postes de président et de trésorier sont des postes particuliers, investis d'un certain pouvoir, tandis que les postes de secrétaires sont moins décisionnaires et plus facilement laissés aux femmes, car souvent proches d'un secrétariat 'classique'» (Tchernonog, 2007, p. 121).

En Espagne, le bénévolat dans le tiers secteur se compose à part égal d'hommes et de femmes avec une légère majorité pour les premiers à l'exception du secteur de l'action sociale où la balance penche nettement du côté des femmes.

Dans le secteur social, largement plus étudié, le profil de la personne volontaire est une femme (57\%) autour de 31 à 45 ans $(45.5 \%)$ avec un niveau d'étude secondaire (43\%), impliquée environ 4h par semaine (Gallego \& Cabrero, 2001). D'autres études évaluent la part des femmes entre 57 et $75 \%$. Le niveau d'étude des volontaires augmente avec la taille de la structure et la prédominance très nette des femmes disparaît dans les organes de direction. Deux études territoriales dans le secteur sanitaire et social apportent des précisions.

Une enquête réalisée dans la Communauté de Valence (Campà, 2004) indique que sur 331 associations d'action sociale interrogées, $40 \%$ sont dirigées par des femmes, avec des variations selon la taille des structures (GTS, 2006), comme en France, et selon le public bénéficiaire. 93\% des ONG dédiés principalement ou exclusivement aux femmes, sont dirigées par des femmes et 47,36 \% des femmes dirigeantes associatives dans ce secteur le sont d'une association visant un public féminin. Suite à ce constat, l'étude pose deux hypothèses d'interprétation, une spécialisation des femmes au détriment de leur présence dans des associations plus généralistes ou une meilleure intégration par ces associations des mesures en faveur de l'égalité des chances dans les associations.

Une seconde enquête, menée sur 18 associations d'appui aux malades et parents du territoire basque et qui perçoivent $70 \%$ du total des subventions en 2001, confirme ce constat. Elle indique que même si les personnes bénéficiaires de ces actions sont à $58 \%$ des femmes, les postes de direction (président, vice-président, secrétaire, trésorier) sont majoritairement occupés par les hommes $(62 \%)$, les hommes étant nettement majoritaires au poste de président et vice-président et les femmes légèrement plus nombreuses au poste de secrétaire et trésorière.

Au Royaume-Uni, la place des femmes dans le paysage associatif est quelque peu différente puisque ces dernières sont plus souvent bénévoles que les hommes ; 64\% des femmes participent à des actions bénévoles contre $54 \%$ des hommes. En outre, elles représentent $58 \%$ des bénévoles récents ( 5 ans au plus) et $54 \%$ des bénévoles de longue date ( 6 ans et au-delà). Le profil de la personne bénévole au Royaume-Uni est une femme âgée de 35-44 ans ou 55-64, occupant un emploi. Il ne nous a pas été possible de trouver des données précises concernant leur place dans les instances de décision, mais un récent article de Sonia Reverter-Bañón, de la London School of Economics, traitant d'aspects plus théoriques sur le genre et la société civile, nous donne une indication plutôt pessimiste : « les relations horizontales entre les citoyens sont le lieu de perpétuelles inégalités, et c'est 
précisément parce que la société civile est un espace non régulé que ces inégalités peuvent s'y perpétuer » (Reverter-Bañón, 2008, p. 10).

\section{La place des femmes dans les associations... la part des salariées}

Les données sur le salariat associatif dont la production est techniquement plus aisée du fait des déclarations fiscales et sociales obligatoires s'avèrent plus précises. Dans les pays ayant connu un taux de chômage élevé, le secteur associatif a constitué une opportunité en termes de création d'emploi qui a suscité l'intérêt des économistes et des politiques. Dans la plupart des pays d'Europe, on constate un mouvement de professionnalisation des associations qui se traduit par une augmentation du salariat. Trois points communs ressortent de l'étude des données sexuées du salariat associatif en France, Grande Bretagne et Espagne : un emploi majoritairement féminin, à des postes d'exécution en particulier dans le social, dans le cadre de contrats à temps partiels.

En France, en 2008, les femmes représentent $68 \%$ des salariés associatifs, tout comme en Grande Bretagne $(69 \%)$ où les deux tiers de l'augmentation nette des emplois associatifs sont attribuables à la création de postes occupés par des femmes (Almond \& Kendall, 2000). Toutefois, le nombre d'emplois à temps partiel est important dans les deux pays ; respectivement $32 \%$ et $40 \%$. En Grande Bretagne, près de la moitié (46\%) des femmes salariées des associations travaillent à temps partiel (21\% des hommes). Parmi elles, $84 \%$ auraient fait le choix du temps partiel, seules $9 \%$ déclarant chercher, sans en trouver, un emploi à temps plein. Mais la nature des postes offerts dans les deux pays diffère sensiblement. En Grande Bretagne, 43\% des emplois associatifs sont très qualifiés, dessinant un type de professionnalisation qui augmente l'attractivité du secteur dans les choix de carrière. Au contraire, en France, les postes de cadres sont plus rares dans le secteur associatif que dans les secteurs privé ou public. Les femmes y ont un peu plus accès, mais dans un ensemble finalement réduit.

Les données sur le secteur de l'action sociale, seules disponibles pour l'Espagne, montrent que les femmes y sont très présentes, $(67,8 \%)$ comme en France $(83 \%)$ (Tchernonog, 2007, p. 17), mais principalement sur les nombreux emplois d'aide à la personne, peu qualifiés. Les femmes y ont des positions salariées moins prestigieuses et de moindre visibilité sociale. On constate une asymétrie dans la distribution du travail et des tâches d'autant plus aiguës dans le secteur social que le contenu des tâches peut être associé aux rôles traditionnels attribués aux femmes concernant les tâches d'exécution dans le travail domestique et du soin. Ces emplois plus fréquemment d'exécution et de service sont aussi souvent à temps partiels.

\section{Associations et pouvoirs publics : quelle responsabilité dans le déséquilibre?}

En France comme en Espagne (GTS, 2006), les enquêtes plus qualitatives font apparaître deux types de discours justifiant ces situations de déséquilibre au regard du genre : les hommes en position dirigeante ou gestionnaire évoquent une collaboration complémentaire et instrumentale ; les femmes parlent d'une division sexuée des tâches conforme aux rôles et identités de genre traditionnels. Dans tous les cas, on note une absence de prise de conscience de ce déséquilibre et du rapport de pouvoir afférent, ainsi qu'un discours de naturalisation de la différence de rapport au pouvoir des hommes et des femmes.

L'accès aux fonctions dirigeantes est un processus qui donne, dans tous les champs du social, une légitimation plus forte aux individus disposant des ressources sociales et 
économiques les plus élevées. Les rapports sociaux traditionnels (CSP, âge, sexe) agissent dans le champ associatif de façon tout aussi efficace que dans les autres domaines de la vie sociale, mais de façon sans doute plus invisible, en raison des principes associatifs tels que la liberté d'engagement et la «bonne volonté ». Le seul rapport qui soit régulièrement évoqué est celui de l'âge, en lien avec la question du renouvellement des générations. En revanche, les rapports sociaux de sexe semblent impensés tandis que les rapports sociaux en termes de CSP seraient quasiment tabou ${ }^{1}$. Ces questions ne sont évoquées en France qu'au niveau national par certaines associations ou fédérations de l'éducation populaire et du mouvement sportif', qui s'emparent de cette question depuis 1999, sous l'impulsion de politiques publiques de promotion de l'égalité. De même, en Espagne, ce n'est que récemment, dans le cadre d'un Forum du tiers secteur, que cette question a été mise en débat dans le champ associatif (Rey, 2008) en référence aux directives européennes de lutte contre les discriminations et dans le prolongement des différentes initiatives et loi sur l'égalité de genre depuis 2004 dans le pays.

Le degré d'implication des associations concernant l'égalité entre les femmes et les hommes est très hétérogène. Certains acteurs jugent ce sujet sans fondement, arguant que l'engagement associatif reposant sur la base du volontariat, les femmes peuvent au même titre que les hommes s'y investir «si elles le souhaitent». D'autres le jugent secondaire, privilégiant la question de l'engagement associatif et du renouvellement des bénévoles sans perspective de genre. En revanche, certaines associations semblent intéressées mais déclarent ne pas savoir comment s'y prendre alors que d'autres, convaincues de l'intérêt de la démarche, s'engagent en théorie et en pratique, voire, dans de plus rares cas, se dotent de moyens pour définir des axes de progrès et élaborent un plan d'action.

\section{Conclusion}

Le champ associatif, loin d'être un havre d'égalité, constitue un terrain comme un autre de (re)production des rapports sociaux de sexe, d'autant plus efficace qu'ils y sont invisibilisés, voire niés. La prise de conscience du problème et l'émergence d'un souci d'égalité y sont pourtant à l'ordre du jour. Elles s'inscrivent dans un contexte global d'évolution vers l'égalité et la mixité à l'œuvre dans le monde économique et politique notamment impulsée par l'Union Européenne ${ }^{3}$. Mais pour que cette problématique puisse passer du questionnement à l'action, il est indispensable que soient construits, de façon rigoureuse et systématique, des données statistiques et des indicateurs sexués pour chaque pays de l'Union Européenne. Ces données harmonisées permettraient en outre de vérifier s'il existe un lien de causalité entre les variantes du monde associatif, telles que nous les avons présentées en début d'article, et la place faite aux femmes dans ces organisations. En l'état actuel des connaissances, il reste hasardeux de conclure dans un sens ou un autre.

\footnotetext{
${ }^{1}$ Lors de la préparation des 1ères Assises nationales de la vie associative en 1999, des forums départementaux ont été organisés afin de faire émerger les thèmes de réflexions et de propositions : 16000 associations ont été représentées, par 250000 participants. La représentation équilibrée des hommes et des femmes n'est évoquée que dans $2 \%$ des débats. La participation des différentes CSP n'est pas évoquée.

${ }^{2}$ Un guide recueille les pratiques d'associations nationales visant à encourager l'égal accès des femmes et des hommes aux responsabilités. (F. Fillon-Nallet, A. Guardiola, L. Nobili, 2004)

${ }^{3}$ Communication de la commission au conseil, au parlement européen, au comité économique et social européen et au comité des régions: Feuille de route pour l'égalité entre les femmes et les hommes, 2006-2010 «Promouvoir la participation des femmes et des hommes sur un pied d'égalité à la prise de décision », 1er mars 2006.
} 


\section{Références bibliographiques :}

Almond Stephen, Kendall Jeremy, 2000, Paid employment in the self-defined voluntary sector in the late 1990s: an initial description of patterns and trends, Centre for Civil Society Working Paper,

Archambault Edith, 2007, «L'économie sociale en France dans une perspective européenne », XXIème colloque ADDES Association pour le Développement de la Documentation sur l'Economie Sociale, 26 novembre, $24 \mathrm{p}$.

Campá García (dir.), novembre 2004 ¿Son machistas las ONG? Primer avance de una investigación socio-jurídica. ww.gts.uji.es/docs/las_palmas.pdf

Clark Jenny, 2007, The UK Voluntary Sector Workforce Almanac 2007, Workforce hub / NCVO

CPCA, 2008, Les secteurs associatifs et leurs relatons avec l'Etat dans l'Europe des 27. Essai d'analyse comparée, CPCA, Coll. «Etudes et documents »

Diebolt Evelyne, 2004, Femmes de conviction, femmes d'action. Les femmes aux postes de décision du secteur associatif (1983-2004), Paris, Femmes et Associations

Equipo de investigación GTS (Género y Tercer Sector), 2006, El «techo de cristal» en las entidades no lucrativas de la Comunidad Valenciana, IX Congreso Estatal del Voluntariado, Universidad Jaume I (Castellón), Toledo

EUROSTAT, 2008, La vie des femmes et des hommes en Europe. Un portrait statistique, Eurostat / Commission Européenne.

http://epp.eurostat.ec.europa.eu/portal/page? pageid $=1073,46587259 \&$ dad=portal\& schema=PORTAL\&p_product code=KS-80-07-135

Fèbvre Michèle, Muller Laura, 2004, Vie associative et bénévolat en 2002. Tableaux issus de l'enquête PCV «Vie associative » et des indicateurs sociaux, INSEE, Série Documents de travail.

Fillon-Nallet Françoise, Guardiola Anne, Nobili Laurence, 2004, Guide expérimental d'appui méthodologique pour l'égal accès des femmes et des hommes aux responsabilités dans les associations, financé par la Délégation Interministériel à l'économie et l'expérimentation sociale, Retravailler

Gallego Marbán, V. y Rodríguez Cabrero, G., 2001, “El voluntariado: prácticas sociales e impactos económicos", en Revista del Ministerio de Trabajo y asuntos Sociales, n¹, (Ejemplar dedicado a: Voluntariado), pp. 49-70

García Delgado, José Luis (dir.), 2004, Las cuentas de la economía social. El tercer sector en España, Madrid, Civita Ediciones

Howell Jude, 2007, “Gender and Civil Society: Time for Cross-Border Dialogue”, Social Politics: International Studies in Gender, State \& Society, vol 14, n4, pp. 415-436

Lorenzo García, Rafael de (dir.), 2003, Tejido asociativo español y tercer sector, Madrid, Editorial Centro de Estudios Ramón Arces, Fundación Luis Vives, DL

Reverter-Bañón Sonia, 2008, "Civil Society and Gender Equality: A Theoretical Approach”, Civil Society Working Paper 24, LSE

Rey Fernando, 2008, Igualdad de trato, de oportunidades y Tercer Sector, éditeur i 
Tabariès Muriel, Tchernonog Viviane, 2005, «Les femmes dans les associations. La non mixité des bureaux, reflet de centres d'intérêt différents ou modalité d'accession aux responsabilités pour les femmes ? », RECMA, $\mathrm{n}^{\circ} 297$, pp. 60-80

Tchernonog Viviane, 2007, L'état des associations. Le paysage associatif et ses évolutions. Activités / Financement / Dirigeants / Emploi Salarié / Travail Bénévole, Juris-Associations - Dalloz 